DOI: $10.12731 / 2306-1561-2013-4-24$

\title{
FORMALIZED DESCRIPTION OF BUSINESS GAME SCENARIO FOR PERSONNEL QUALIFICATION EVALUATION
}

\author{
Nikolaev A.B., Barinov K.A.
}

\section{Abstract}

The article analyzes the game-theoretic model of non-cooperative interaction between several agents. It is assumed that agents make decisions independently and simultaneously, without being able to agree on the selected action to redistribute the resulting utility (win), etc.

The mathematical models and methods of formalized description of the simulation game scenario are considered. The place and the role of business games in the process of evaluating the qualifications of the personnel of enterprises are revealed. Formal method of qualification evaluation is proposed.

Keywords: business game, agent, parallel processes, multi-role game, qualifications of the personnel, the player.

\section{УДК 681.3}

\section{ФОРМАЛИЗОВАННОЕ ОПИСАНИЕ СЦЕНАРИЯ ДЕЛОВОЙ ИГРЫ ПО ОЦЕНКЕ КВАЛИФИКАЦИОННЫХ ХАРАКТЕРИСТИК ПЕРСОНАЛА}

\section{Николаев А.Б., Баринов К.А.}

\section{Аннотация}

В статье проведен анализ теоретико-игровой модели некооперативного взаимодействия между несколькими агентами. Предполагается, что агенть принимают решения одновременно и независимо, не имея возможности договариваться о выбираемых действиях, перераспределять получаемую полезность (выигрыи) и т.д.

Рассмотрены математические модели и методы формализованного описания сценария деловой игры. Показано место и роль деловых игр в процессе оценки квалификационных характеристик персонала предприятий. Предложена методика оценки квалификации.

Ключевые слова: деловая игра, агент, параллельные процессы, многоролевая игра, квалификачия персонала, игрок. 


\section{Теоретико-игровая модель некооперативного взаимодействия агентов}

Каждый агент осуществляет выбор действия хі, принадлежащего допустимому множеству $X_{i}, \quad i \in N=\{1,2, \ldots, n\}-$ множеству агентов. Выбор действий агентами осуществляется однократно, одновременно и независимо.

Выигрыш і-го агента зависит от его собственного действия хi $\in \mathrm{Xi}$, от вектора действий

$$
x_{-\mathrm{i}}=\left(x_{1}, x_{2}, \ldots, x_{i-1}, x_{i+1}, \ldots, x_{n}\right) \in X_{-i}=\prod_{j \in N \backslash\{i\}} X_{j}
$$

оппонентов $N\{i\}$ и от состояния природы $\theta \in \Omega$, и описывается действительнозначной функцией выигрыша $f_{i}=f_{i}(\theta, x)$, где $x=\left(x_{i}, x_{-i}\right)=\left(x_{1}, x_{2}, \ldots, x_{n}\right)$ $\in \mathrm{X}^{\prime}=\prod_{j \in N} X_{j}-$ вектор действий всех агентов. При фиксированном значении состояния природы совокупность $\Gamma=\left(N,\left\{X_{i}\right\} i \in N,\left\{f_{i}(\bullet)\right\} i \in N\right)$ множества агентов, множеств их допустимых действий и целевых функций представляет игру в нормальной форме. Решение игры в данной модели (равновесие) представляет множество устойчивых в том или ином смысле векторов действий агентов.

Предполагается, что каждый агент будет стремиться выбрать наилучшие для него (с точки зрения значения его целевой функции) действия при заданной обстановке. Обстановкой для него будет совокупность состояния природы $\theta \in \Omega$ и обстановки игры $x_{-\mathrm{i}}=\left(x_{1}, x_{2}, \ldots, x_{i-1}, x_{i+1}, \ldots, x_{n}\right) \in X_{-i}=\prod_{j \in N \backslash\{i\}} X_{j}$.

Принцип принятия им решения о выбираемом действии (при фиксированных обстановке и состоянии природы) в данной модели определяется следующим образом:

$$
B R_{\mathrm{i}}\left(\theta, x_{-i}\right)=\arg \max _{x_{i} \in X_{i}} f_{\mathrm{i}}\left(\theta, x_{i}, x_{-i}\right), i \in N
$$

Адекватным математическим инструментом моделирования связей между объектами являются графы. В рамках рассматриваемых моделей сетевого взаимодействия вершинами графа являются игроки, а дуга интерпретируется как наличие направленной связи между игроками.

Пусть имеется множество игроков $N=\{1, \ldots, n\}$, каждый из которых обладает некоторой информацией. Игрок может сообщать имеющуюся у него информацию другим (возможно, не всем) игрокам. Тогда структура сообщений будет описываться конечным графом, вершинами которого являются игроки, а дуга ij содержится в графе тогда и только тогда, когда игрок і сообщает некоторую информацию игроку ј. Если обозначить через $\Theta(N)$ множество всех графов с множеством вершин $\mathrm{N}$, то заинтересованность і-го игрока в той или иной структуре связей можно описать функцией выигрыша $f_{i}: \Theta(N) \rightarrow \Re, \quad i \in N$, определяющей выигрыш игрока при реализации различных структур. 
Таким образом, в модель сетевой игры входят множество игроков $N=\{1, \ldots, n\}$ и набор функций выигрыша $f_{i}: \Theta(N) \rightarrow \Re, i \in N$.

Пусть в условиях предыдущего примера выигрыш, который $i$-й игрок получает от сообщения $j$-го игрока, равен $p i j$, а затраты $j$-го игрока по передаче сообщения $i$-му игроку равны $c_{j i}$. Игрок, получивший информацию от другого игрока, может передавать ее далее по сети. Введем коэффициент искажения информации $a \in(0,1)$ при передаче ее по одной дуге. Тогда выигрыш $i$-го игрока $f_{i}(g)$ в сети $g=\langle N, E\rangle \in \Theta(N)$ можно записать в виде:

$$
f_{i}(g)=\sum_{j \in N, j \neq i} p_{i j} \alpha^{\delta_{g}(j, i)}-\sum_{j: i j \in E, j \neq i} c_{i j}
$$

где $\delta g(j, i)$ - длина кратчайшего направленного пути в графе g от игрока $j$ к игроку $i$ (если путь отсутствует, считаем, что $\delta g(j, i)=+\infty)$.

Считается, что игроки могут каким-либо образом воздействовать на формирование определенных связей сети. При этом их роль в процессе формирования связей может быть более сложной, чем показано в приведенных примерах, где образование связи $i j$ зависело только от желания $i$-го игрока.

Во многих сетевых играх для образования связи необходимо согласие обоих игроков - например, игрок по каким-либо причинам может отказаться принимать информацию от другого игрока. Итак, чтобы модель сетевой игры стала стратегической моделью, необходимо формализовать возможности игроков по образованию связей.

Желание $i$-го игрока образовать связь іј можно описать переменной xijout, которая равна единице, если игрок і хочет образовать связь ij, и нулю в противном случае. Индекс «out» при переменной показывает, что связь ij по отношению к игроку $i$ является исходящей. Если $x_{i j o u t}$ равна единице, будем говорить, что игрок $i$ имеет предложение к игроку $j$.

Аналогично можно определить переменную $x_{i j i n}$, говорящую о том, что игрок $i$ согласен на образование дуги $j i$ от игрока $j$. Индекс «in» говорит о том, что дуга $\mathrm{ji}$ является входящей по отношению к игроку $i$. Если $x_{i j i n}$ равно единице, будем говорить, что игрок $i$ принимает предложение игрока $j$.

Действие $x_{i}$ игрока $i$ в сетевой игре представляет пару $x_{i}=\left(x_{i o u t}, x_{i i n}\right)$ векторов $x_{\text {iout }}=\left(x_{\text {ilout }}, \ldots, x_{\text {inout }}\right), x_{\text {iin }}=\left(x_{\text {liin }}, \ldots, x_{\text {niin }}\right)$. Множество всевозможных пар этих векторов обозначим через $X_{i 0}$.

Таким образом, действие игрока определяет, по сути, множество его оппонентов, к которым игрок хочет образовать исходящую связь, и множество оппонентов, на образование входящей связи от которых игрок согласен.

Тогда в каждой конкретной игре множество $X_{i}$ допустимых действий $i$-го игрока будет подмножеством множества $X_{i 0}$. 
Профиль действий игроков в сетевой игре представляет пару $\mathrm{x}=$ (xout, xin) квадратных матриц размера n, элементами которых являются компоненты допустимых xijout, xijin действий игроков. Множество профилей действий сетевой игры будет представлять декартово произведение $X=\prod_{i \in N} X_{i}$.

Обстановка $x$ - $i$ для $i$-го игрока представляет пару $x$-i $=(x$-iout, $x$-iin) матриц размера $n \times(n-1)$, элементами которых являются компоненты допустимых действий xijout, xijin всех игроков, кроме $i$-го. Профиль действий х складывается из действия игрока хі и его обстановки $x$ - $i$, в этом случае будем считать, что $x=\left(x_{i}, x-i\right)$.

Пусть реализовался некоторый профиль действий $x=\left(x_{o u t}, x_{i n}\right)$. Тогда, если мы считаем, что для образования связи $i j$ необходимо и достаточно согласия обоих игроков, результирующая сеть $g$ определяется поэлементным умножением матрицы xout на транспонированную матрицу $x_{i n}$, т.е. $g=x_{\text {out }} \otimes x_{i n} T$. Множество сетей, достижимых при заданном множестве профилей действий $X$, обозначим через $G(X)$.

В результате стратегическую модель сетевой игры можно определить как совокупность $\left\langle N, f_{i}, X_{i}, i \in N>\right.$ - множества игроков $N$, их функций выигрыша $f_{i}(\cdot)$, $i \in N$ и множеств допустимых действий $X i \subseteq X_{i 0}, i \in N$.

\section{Формализованное описание деловой игры}

Деловая игра наряду с другими методами обучения служит накоплению управленческого опыта, близкого к реальному. Причем с помощью деловых игр это удается сделать несколько лучше, чем при других методах познания [1 - 15]. Игра, вопервых, достаточно реально имитирует существующую действительность; во-вторых, создает динамичные организационные модели; в-третьих, более интенсивно побуждает к решению намеченных целей.

Указанные обстоятельства определяют актуальность использования деловых игр в процессе обучения персонала и оценки их квалификационных характеристик.

Для организации и проведения компьютерных деловых игр необходимо [10 - 13]:

- подготовить руководство игры и группы обеспечения;

- подготовить методическое и техническое обеспечение;

- провести адаптацию деловой игры к соответствующему контингенту участников и условиям ее проведения;

- подготовить будущих участников игры, оценить уровень их готовности к игре;

- выполнить необходимые расчеты для оценки последствий различных вариантов решений, сформировать оптимальное или рациональное решение для каждого фрагмента игры.

Метод анализа конкретных ситуаций, заключается в том, что обучаемым предъявляется ситуация, связанная с некоторым моментом функционирования конкретной системы. Задача обучаемых - коллективное принятие управленческого 
решения в конкретной ситуации. Характерологическим признаком анализа конкретной ситуации являются:

- наличие модели реальной системы, состояние которой рассматривается в некоторый дискретный момент времени;

- коллективная выработка решений;

- многоальтернативность решений;

- единая цель группы при выработке решений;

- наличие системы группового оценивания деятельности обучаемых.

- наличие управляемого эмоционального напряжения обучаемых.

Применение метода анализа конкретных ситуаций целесообразно в тех случаях, когда рассматривается отдельная, относительно сложная организационная, экономическая или управленческая задача, единственно правильное решение или правильное решение которой заранее известно преподавателю. За ним остается последнее слово при подведении итогов.

\section{Ролевые игры}

В таких играх обычно отрабатывается умение руководить людьми. Для проведения игры необходимо моделировать управляющие системы, входящие в структуру конкретных систем. Среди участников игры при разборе предлагаемых ситуаций распределяются роли, которые могут носить групповой характер. Задача игры: выработка для исполнителя каждой роли на каждом игры оптимальной стратегии поведения.

В отличие от анализа конкретных ситуаций в ролевых играх:

- необходимо наличие модели управляющей системы;

- решение принятое обучаемым воздействует только на управляющую систему, а не на объект управления;

- комплекс-модель управляющей системы носит динамический характер, где есть ролевое общение, в котором общение может зависеть от хода общения между партнерами.

Имитационные игры - это игры с разной целевой направленностью, для которых не свойственны некоторые из перечисленных характерологических признаков ролевых игр.

В такой игре может быть только одна роль, которая тиражируется (создается множество экземпляров) каждым участником, который в свою очередь готовит свое решение.

Отсутствует модель управленческой системы и объект управления, остается только модель среды, в которой необходимо принять решение. Здесь моделируется деятельность конкретных руководящих работников или специалистов.

Отсутствие конфликтной ситуации. 


\section{Организационно-деятельностные игры (ОДИ)}

К классу этих игр можно отнести: проблемные ролевые игры, проблемноориентированные деловые игры, апробационно-поисковые игры, инновационные игры. ОДИ применяют при решении сложных социально-производственных задач, когда требуется объединение усилий специалистов разных направлений. Игра базируется на основе предъявляемой игрокам исходной информации о состоянии реальной системы. Далее идет выработка, обсуждение и принятие решений по управлению указанной системой. В таких играх чаще решаются вопросы не оперативного управления, а о разработке программы управления. В игре выявляется комплекс проблем по управлению социально-экономической системой и пути решения этих проблем. В ОДИ обычно рассматриваются кризисные для системы состояния, обеспечивающие повышенную мотивацию участников игры и выработке решений, выводящих систему из кризиса.

Представляет интерес подход, в рамках которого осуществляется переход от игр, в которых участники выбирают свои действия одновременно (игра $\Gamma_{0}$ в нормальной форме), к иерархическим играм, в которых последовательность ходов фиксирована первым делает ход центр, а затем - участник. Можно усложнять модель и дальше, переходя к всё более сложным играм. Проведем логику перехода от более простых задач, к более сложным задачам (рисунок 1), чтобы более сложная задача могла быть декомпозирована на более простые задачи.

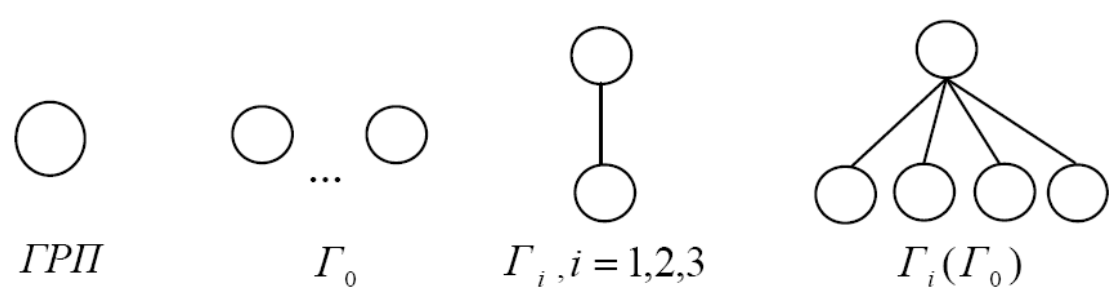

a)

б)

B)

г)

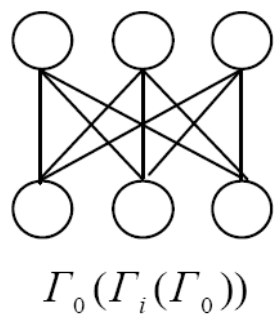

д)

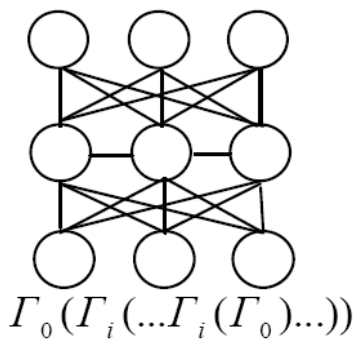

e)

\section{Рисунок 1 - Структуры деловых игр}

Если имеется один субъект, принимающий решения (рисунок 1, a), то он описывается с точки зрения гипотезы рационального поведения как стремящийся максимизировать свою целевую функцию. Далее можно усложнить модель и рассмотреть несколько субъектов на одном уровне (рисунок 1, б), описав их 
взаимодействие игрой $\Gamma_{0}$ в нормальной форме. Если ввести иерархию, то для двух субъектов (рисунок 1, в) их взаимодействие описывается игрой $\Gamma_{i}$, где $i \in\{1,2,3\}$.

Предположим, что имеется структура «один начальник - несколько подчиненных» (рисунок 1, г). Взаимодействие участников, находящихся на одном уровне, можно описывать игрой ГО. Взаимодействие «начальник-подчиненный» описывается игрой $\Gamma_{i}$. Тогда условно такую структуру можно представить игрой $\Gamma_{i}$, определенной на игре $\Gamma_{0}$, условно обозначив ее $\Gamma_{i}\left(\Gamma_{0}\right)$. Далее, пусть есть несколько начальников (центров) и несколько подчиненных (рисунок 1, д). На нижнем уровне участники играют игру $\Gamma_{0}$. Над ними центры играют иерархическую игру $\Gamma_{i}$, но центры, в свою очередь, разыгрывают на своем уровне игру $\Gamma_{0}$. В результате получим игру $\Gamma_{0}\left(\Gamma i\left(\Gamma_{0}\right)\right)$. Можно взять более сложную структуру с более сложным взаимодействием (рисунок 1 , е). Это будет иерархическая игра между уровнями, и "обычная" игра на каждом из уровней: $\Gamma_{0}\left(\Gamma_{i}\left(\ldots \Gamma_{i}\left(\Gamma_{0}\right) \ldots\right)\right.$.

Основная идея заключается в том, чтобы декомпозировать сложную структуру игры на набор более простых структур и воспользоваться результатами исследования последних. Кроме того, между играми и структурами существует глубокая связь момент принятия субъектом решений определяет его "место" в организационной иерархии.

Несомненно, полезно иметь такие механизмы формирования сценариев, когда несколько игр естественным образом объединяются для решения более крупной задачи, в том числе, и моделирования производственной ситуации.

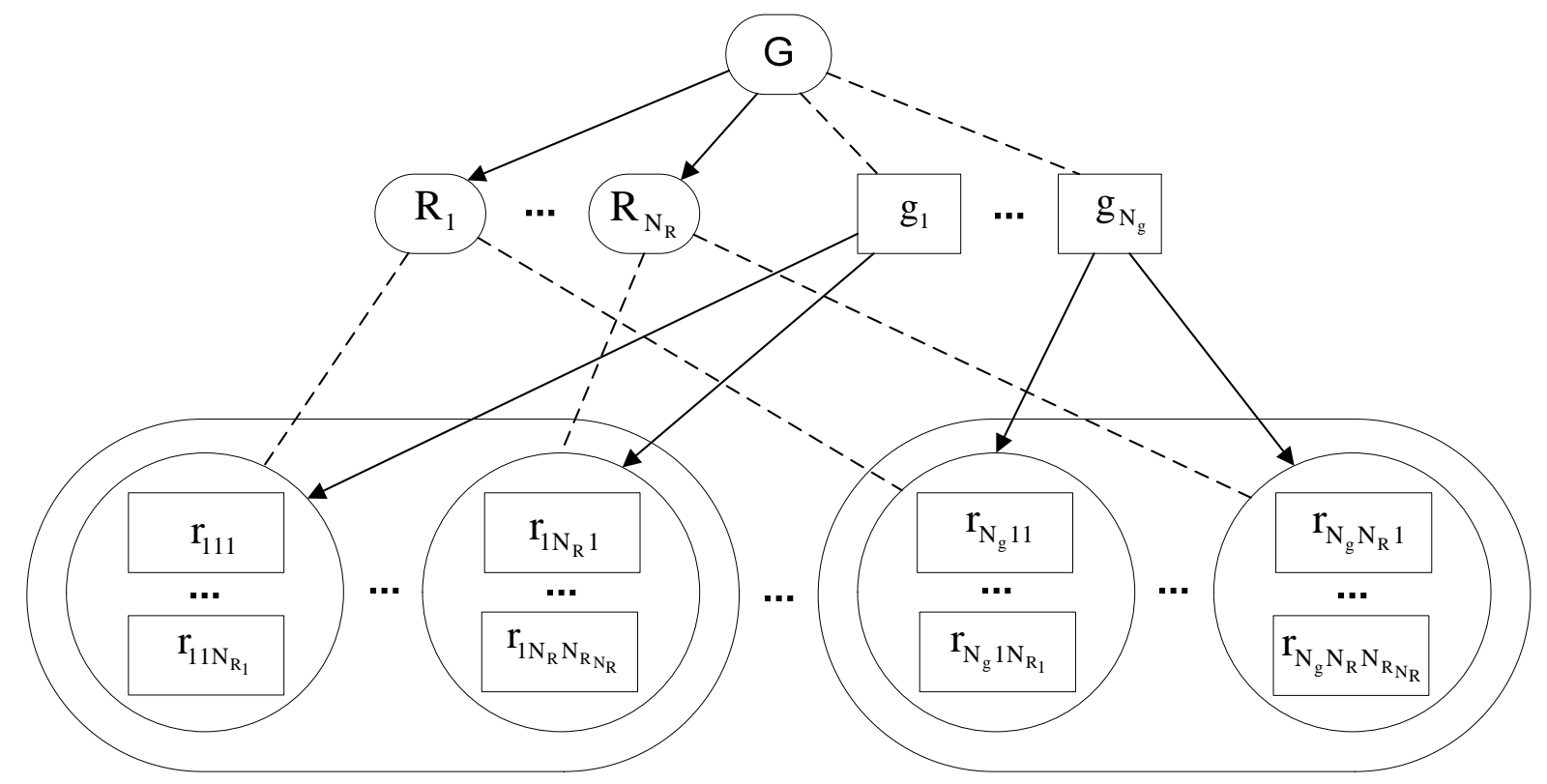

Рисунок 2 - Общий случай организационно-структурной среды многоролевой деловой игры 
Предлагается использовать многоуровневый подход к описанию сценариев, которые получил программную поддержку в системе «СОТА». Общий случай организационно-структурной среды многоролевой ДИ представлен на рисунок 2, где:

$G$ - многоролевая деловая игра (МРДИ);

$R_{j}$ - роли, предусмотренные в игре $G ; 1 \leq j \leq N R$, где $N R$ - количество ролей, предусмотренных в игре $G$;

$g_{i}$ - созданные экземпляры игры $G ; 1 \leq i \leq N_{g}$, где $N_{g}$ - количество созданных экземпляров игры $G$;

$r_{i j k}$ - экземпляры предусмотренных ролей в созданных экземплярах игры $G ; 1 \leq$ $k \leq N_{R_{j}}$, где $N_{R_{j}}$ - число созданных экземпляров роли $R_{j}$.

По своей структуре индивидуальная одноэкземплярная ДИ, имеющая возможность создания нескольких взаимодействующих экземпляров своей единственной роли, аналогична индивидуальной ДИ с единственным экземпляром этой роли, но поддерживающая несколько взаимодействующих экземпляров самой игры.

Пунктирными линиями показаны отношения типа «класс-экземпляр». Стрелками показаны отношения принадлежности. При разработке тела ДИ в сценарии возможны различные варианты организации потоков управления (рисунок ЗОшибка! Источник ссылки не найден.). Восьмиугольниками обозначены синхронизующие фрагменты.

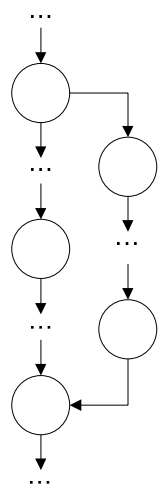

a)

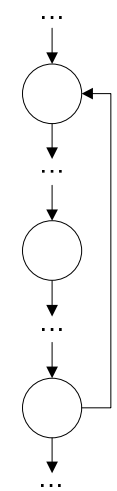

б)

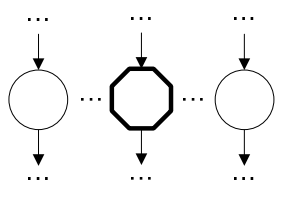

B)

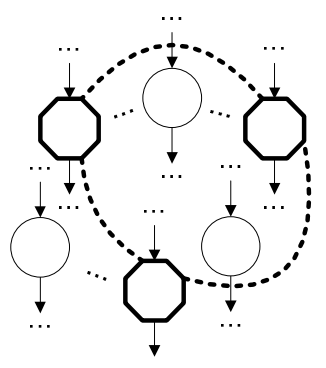

г)

\section{Рисунок 3 - Варианты организации потоков управления}

Пунктирной линией показан контур синхронизации, объединяющий фрагменты, относящиеся к разным ролям (нитям сценария). Синхронизироваться будут экземпляры только тех нитей, которые входят в контур. Фрагменты рисунка соответственно определяют:

а) ветвление;

б) цикл;

в) синхронизация всех экземпляров в пределах одной роли; 
г) синхронизация всех экземпляров всех ролей, входящих в синхронизирующий контур.

Функционирование каркаса ДИ определяется следующими параметрами, значения которые задаются при разработке игры: экземплярность ДИ: одно- или многоэкземплярная; ограничения на выбор ролей.

Формализация представления сценария действий пользователей в ходе деловой игры для его последующей программной реализации

Для эффективной программной реализации действий пользователей в рамках единой деловой игры предлагается организация параллельного выполнения процессов в пределах одного экземпляра проигрывателя ДИ.

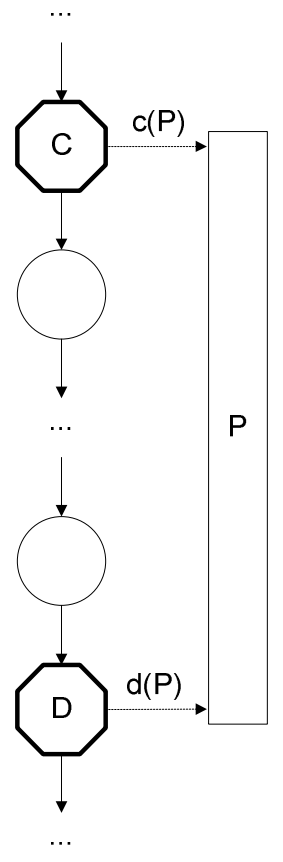

a) с принудительным уничтожением фонового процесса

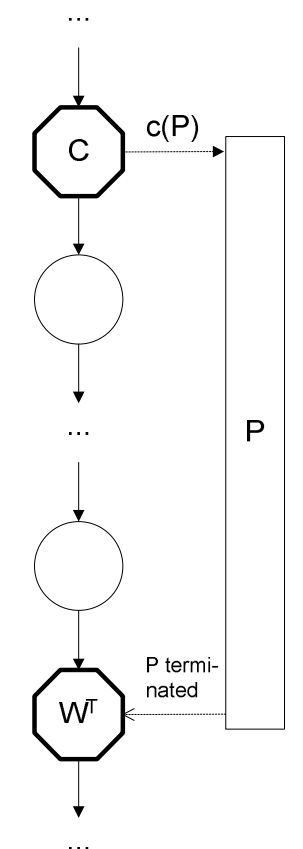

б) с ожиданием самостоятельного завершения фонового процесса

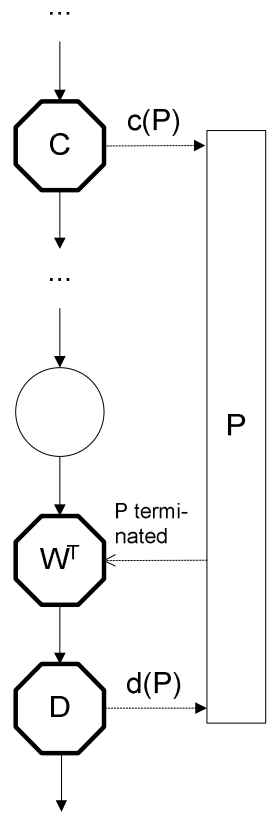

в) комбинированный вариант завершения

\section{Рисунок 4 - Организация параллельных пользовательских процессов}

Для поддержки взаимодействующих параллельных процессов в сценарий вводятся дополнительные фрагменты, играющие вспомогательную роль, 3-х типов (рисунок 4):

- создающие $(\mathrm{C})$ - создают фоновый процесс;

- уничтожающие (D) — принудительно уничтожают фоновый процесс (рисунок 4, a); 
- ожидающие (WE) - блокируют выполнение основной нити сценария в ожидании наступления некоторого события, связанного с выполнением фонового потока. Их частный случай (WT) - ожидание самостоятельного завершения фонового процесса.

Для ожидающего фрагмента также может быть задано максимальное время ожидания. Тогда ожидающий фрагмент блокирует выполнение основной нити сценария до момента наступления заданного события, связанного с выполнением фонового процесса, либо до истечения максимального времени ожидания (рисунок 4, б). Наступление события связано с изменением состояния некоторого ресурса, которым может быть сам фоновый процесс или любой другой объект.

Эти дополнительные фрагменты также являются невизуальными (не имеют визуального представления). Фоновые процессы существуют не дольше времени проигрывания структурного элемента, в контексте которого он был порожден. При создании фонового процесса его идентификатор помещается во внутренний список фоновых процессов породившего его экземпляра проигрывателя. Этот список используется для принудительного уничтожения экземпляром проигрывателя фоновых процессов, которые на данный момент времени уже не должны существовать, но по каким-либо причинам не были уничтожены соответствующими фрагментами сценария структурного элемента.

$P_{k}, P_{m}-$ фоновые процессы. Пунктирными стрелками с закрашенным наконечником показаны управляющие действия, связанные с созданием и уничтожением фоновых процессов. Пунктирными стрелками с наконечником в виде уголка показаны сообщения от фоновых процессов, сигнализирующие о наступлении ожидаемого события. Жирными стрелками показано взаимодействие между фрагментами основной нити сценария и фоновыми процессами, а также взаимодействие фоновых процессов между собой.

Фрагменты основной нити сценария могут взаимодействовать с фоновыми процессами посредством: СОМ-интерфейсов; общей памяти; именованных каналов; файлов; баз данных; других разделяемых объектов. Возможен комбинированный вариант завершения фонового процесса (рисунок 4, в), при котором в течение заданного периода времени ожидается самостоятельное завершение фонового процесса. Если за указанный интервал времени процесс не завершился, то он завершается принудительно.

В виде фоновых процессов имеет смысл организовывать невизуальные процессы, выполняющие вспомогательные, обслуживающие функции для основной нити сценария. В основной нити сценария реализуются элементы пользовательского интерфейса, которые могут взаимодействовать с фоновым процессом. Использование параллельных процессов в пределах одного экземпляра проигрывателя ДИ способствует формированию более компактного сценария и его более эффективному в плане организации во времени выполнению. 


\section{Идентификация уровня подготовки персонала в ходе проведения деловых игр}

Для реализации процедур идентификации уровня подготовки персонала в ходе проведения деловых игр, в работе предложена итерационная процедура. При этом определение квалификационного уровня определенного сотрудника является отдельной задачей и ее решение может быть сведено к обработке экспертных оценок всех остальных сотрудников.

Пусть имеется $N$ участников деловой игры, в процессе которой либо вручную, либо автоматически (анализ действий в соответствии со сценарием) каждый дает свою оценку “квалификационного уровня” другому, что может быть сведено в матрицу $X$, где $x_{i j}$ - оценка $i$-ым участником “квалификации” $j$-го участника. Интересен вопрос согласованности этих оценок. При полном согласовании все строки должны совпадать.

Однако в общем случае строки не совпадают за счет субъективного мнения каждого. Наиболее естественным путем получения результирующей оценки является взвешенная оценка всех участников:

$$
X i=\alpha 1 \cdot x 1 i+\alpha 2 \cdot x 2 i+\ldots+\alpha N \cdot x N i
$$

где $\alpha_{i}-$ истинная оценка участника.

Однако истинной оценкой будем считать ту, которую получим после преобразования с учетом всех построенных оценок. В результате получаем итерационную процедуру:

$$
X^{(k)}=\sum_{i=1}^{N} x_{i j} \cdot X_{i}^{(k-1)}
$$

В качестве начальных весов можем взять веса $X(0)$, определенные некоторым одним случайно или неслучайно выбранным участником. После этого взвесим оценку каждого участника этими весами, получим новые веса каждого и т.д.

\section{Заключение}

В работе рассмотрена теоретико-игровая модель некооперативного сетевого взаимодействия участников многоролевой деловой игры.

Проведен анализ и классификация деловых компьютерных игр в системе переподготовки персонала промышленных предприятий. Показана целесообразность использования организационно-деятельностных и многоролевых игр.

Предложен многоуровневый подход к описанию сценариев многоролевых деловых игр, основанный на декомпозиции игр со сложной структурой до игр с простой структурой и построения соответствующей иерархии.

Разработаны механизмы синхронизации действий пользователей в процессе многоролевой деловой игры и предложена методика оценки и переоценки квалификации персонала по результатам проведения деловой игры, что позволило разработать программные компоненты расширения функционала программноинструментальной среды конструирования многоролевых деловых игр. 


\section{Список информационных источников}

[1] Баринов К.А., Власов А.Б., Строганов В.Ю., Ягудаев Г. Г. Формирование организационной структуры управления промышленным предприятием с использованием многоролевых деловых игр // Наука и образование. МГТУ им. Н.Э. Баумана. Электрон. журн. 2011. № 8. Режим доступа: http://technomag.edu.ru/doc/206805.html

[2] Баринов К.А., Рачковская В.М., Солнцев А.А., Тимофеев П.А. Инструментарий деловых игр в задачах управления реализацией продукции в условиях конкуренции // Наука и образование. МГТУ им. Н.Э. Баумана. Электрон. журн. 2011. № 9. Режим доступа: http://technomag.edu.ru/doc/207618.html

[3] Баринов К.А., Горячкин Б.С., Иванова Л.В., Николаев А.Б. Формальные модели представления и организации деловых игр // Наука и образование. МГТУ им. Н.Э. Баумана. Электрон. журн. 2011. № 9. Режим доступа: http://technomag.edu.ru/doc/207391.html

[4] Пугачев В.П. Тесты, деловые игры, тренинги в управлении персоналом. - М., 2002.

[5] Платов В.Я. Деловые игры: разработка, организация, проведение. - М., 1991.

[6] Гуреев А.Б., Динова Н.И., Кулжабаев Н.М., Щепкин А.В. Учебные автоматизированные деловые игры. - М.: ИПУ им. В.А. Трапезникова РАН, 1999.

[7] Петросян Л.А., Зенкевич Н.А., Семина Е.А. Теория игр. - М.: Высшая школа, 1998.

[8] Новиков Д.А., Чхартишвили А.Г. Рефлексивные игры. - М.: Синтег, 2003. - 160 с.

[9] Губко М.В., Новиков Д.А. Теория игр в управлении организационными системами. - М.: Синтег, 2002. - 148 с.

[10] Остроух А.В. Реализация деловых игр в компьютерных системах обучения / К.А. Баринов, А.В. Остроух, Н.Е. Суркова // Открытое и дистанционное образование. Томск: ОАО «Издательство ТГУ», 2007. - №3 (27), - С. 28-33.

[11] Остроух А.В. Реализация деловых игр в компьютерных системах обучения / К.А. Баринов, А.В. Остроух, Н.Е. Суркова // Ученые записки ИИО РАО. - М.: ФГНУ "Институт информатизации образования" Российской академии образования, 2007. - Вып. 24. - С. 40-50.

[12] Баринов К.А. Опыт разработки и использования ролевых игр для подготовки и переподготовки специалистов предприятий промышленности и транспортного комплекса / К.А. Баринов, Д.А. Буров, А.В. Бугаев, А.В. Остроух // Научный вестник МГТУ ГА. - 2009. - №141. - С. 188-197.

[13] Остроух А.В. Информационные технологии в научной и производственной деятельности / [ред. А.В. Остроух] - М: ООО "Техполиграфцентр", 2011. - 240 с. ISBN 978-5-94385-056-1.

[14] Исмоилов М.И. Подготовка и переподготовка персонала предприятий промышленного и транспортного комплексов с применением мобильных технологий: Монография / М.И. Исмоилов, А.Б. Николаев, А.В. Остроух. - SaintLouis, MO, USA: Publishing House Science and Innovation Center, 2013. - 166 c. ISBN 978-0-615-67111-6.

[15] Остроух, А.В. Опыт разработки электронных образовательных ресурсов нового поколения для дистанционной технологии обучения / А.В. Остроух // В мире научных открытий. - 2011. - №9 (21). - С.149-158. 